\title{
Optimization of a neutron dosimeter for the high energy accelerators
}

\author{
Alexey Sokolov ${ }^{1, \star}$, Georg Fehrenbacher ${ }^{1}$, Ekaterina Kozlova ${ }^{1}$, Torsten Radon ${ }^{1}$, and Izabella Zahradnik $^{1}$ \\ ${ }^{1} \mathrm{GSI}$, Darmstadt (Germany)
}

\begin{abstract}
In high energy accelerator facilities the neutron radiation should be continuously measured during operation to control the ambient dose. This requires a reliable neutron dosimeter in a wide energy range. In this work we present an optimization of a compact cylindrical passive neutron dosimeter for the usage in wide energy neutron fields.
\end{abstract}

\section{Introduction}

For various high energy physics experiments, ions are accelerated to high energies and in collision with a target or losses in a beam line produce neutrons. These neutron fields are possibly harmful not only to the personnel behind the shielding, but also to electronics located in the vicinity of a radiation source. The energy range of neutrons at accelerator complexes, such as the future Facility for Anti-proton and Ion Research (FAIR) [1] can vary from thermal up to GeVs. The position of the ion losses in the beam line are often unpredictable and time dependent thus an accumulative dose measurement of the ambient dose is preferred for monitoring over a wide area. This and necessity in a reliable measurements during a long period, for instance one year, can be provided with thermoluminescence detector (TLD) based dosimeters [2, 3]. In the TLD cards the neutrons are detected via a ${ }^{6} \operatorname{Li}(n, \alpha)^{3} \mathrm{H}$ reaction whose cross section increases for low energy neutrons. To perform neutron fluence to equivalent ambient dose conversion an appropriate combination of a neutron moderator and absorber layouts should be used. In this work we present the results of simulations and test measurements of a recently developed compact dosimeter layout [4] and continue to search for new materials and layouts for further improvements. The choice of materials and dimensions of different layers were tested with simulations using the FLUKA code [5], results were processed using FLAIR [6].

\section{Dosimeter reading calculations}

The TLD cards signal is proportional to the absorbed dose in the TL chips. In the simulations the energy absorbed in TL chips under irradiation in isotropic neutron fields was scored. The fields used are simple parallel neutron beams of certain energy and additionally a ${ }^{241} \mathrm{AmBe}$ source spectrum which was also used for calibration purposes. The

\footnotetext{
^e-mail: Al.Sokolov@gsi.de
}

TLD card of Harshaw type consists of two pairs of chips, 'TLD-600' based on ${ }^{6} \mathrm{Li}$, sensitive to gamma and neutron radiation and 'TLD-700' based on ${ }^{7} \mathrm{Li}$, sensitive to the gamma radiation. So the simulated relative reading or response of the dosimeter to a certain neutron energy was obtained as a sum of the absorbed dose in the TLD-600 crystals minus the dose in the TLD-700 crystals and divided by the same value for the neutrons of a ${ }^{241} \mathrm{AmBe}$ source spectrum. The calculated relative reading is further compared to the fluence-to-dose values according to Pellicioni's publication [7] normalized to $391 \mathrm{pSv} \cdot \mathrm{cm}^{2}$, which is a reference for ${ }^{241} \mathrm{AmBe}$ source provided by ISO Standard 8529-1 [8]. The closer are the relative readings to the mentioned values, the closer are the ambient dose readings of the dosimeter to the real equivalent dose $\mathrm{H}^{*}(10)$.

FLUKA is also capable to predict an amount of produced isotopes in reactions of neutrons with matter. Several simulations were performed to count the number of ${ }^{3} \mathrm{H}$ and $\alpha$-particles produced in TLD-600 chips during the detection time and the relative dosimeter reading was obtained as:

$$
R_{R}=N_{E}\left({ }^{3} H, \alpha\right) / N_{A m B e}\left({ }^{3} H, \alpha\right)
$$

where $N_{E}\left({ }^{3} H, \alpha\right)$ is either the number of produced tritium or helium nuclei for a certain neutron energy $E$ and $N_{A m B e}\left({ }^{3} H, \alpha\right)$ is the number of those produced under irradiation with neutrons of a ${ }^{241} \mathrm{AmBe}$ spectrum. Such an approach provided the same response characteristics as in the first method, which takes into account the absorbed energy. This shows that the neutron capture process releases the energy which contributes mostly to the absorbed dose in the TL chips even for fast neutrons.

The method calculating the amount of e.g. ${ }^{3} \mathrm{H}-$ particles is very suitable to estimate the dose reading of active detectors, where the dose is calculated as a number of pulses. Each pulse corresponds to a neutron capture event. As a couple of tests we have also performed some simulations for active ${ }^{3} \mathrm{He}$ dosimeters where neutrons are detected in the ${ }^{3} \mathrm{He}(n, p)^{3} \mathrm{H}$ reaction. Again we used also the 
absorption energy for detection and as usually both methods of isotope counting and energy deposition calculations gave the same response characteristics of the investigated dosimeter layouts.

The energy dependence of the capture cross sections for ${ }^{3} \mathrm{He}$ and ${ }^{6} \mathrm{Li}$ are pretty similar and our simulations showed that exchange of a TLD card with a ${ }^{3} \mathrm{He}$ proportional counter is possible. Exchanging the sensitive element of the dosimeter in studied layouts should provide close relative read-out. Of course the response characteristics are influenced by the size of the ${ }^{3} \mathrm{He}$ tube as well as by the gas pressures of the counter. The active dosimeter prototypes are to be tested in the near future.

\section{Compact cylindrical dosimeter}

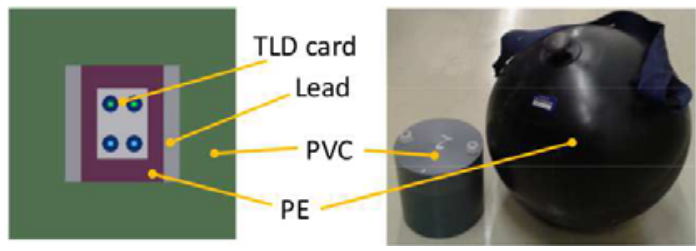

Figure 1. (left) Neutron dosimeter layout with a sensitive element - TLD card, PVC, lead and PE layers. Upper green squares in the TLD card depict TLD-700 chips and lower blue ones TLD600 chips. (right) Prototype 2 of the dosimeter and the 12" sphere [3] currently used at GSI for ambient dose measurements.

As it was shown by us previously [4, 9] compact sizes of broad energy range neutron dosimeters are possible, introducing a polyvinylchloride (PVC) layer, which serves for both absorption and moderation. This material also reduces the production costs and makes the dosimeter design simple. In Fig. 1 (left) one can see a schematic layout of a cylinder dosimeter and a photo of a built prototype Fig. 1 (right). The dosimeter has an outer PVC layer, afterwards a lead layer used to increase the response for high energy neutrons [3]. In the middle are an additional moderating high density polyethylene (PE) layer and the TLD card, as a sensitive element. The developed design with height and diameter of only $14 \mathrm{~cm}$ and weight of 4.5 kilogram provided a very good response of the dosimeter in high energy region (MeVs - GeVs) critical at high energy accelerators. Still the over-response in medium (eVs-keVs) energy region leads to a dose overestimation. The relative dosimeter reading calculated using FLUKA simulations can be found in Fig. 2. In further sections we describe a possibility to improve the response in the eVs-keVs energy interval. This is necessary even if the measured neutrons are of higher energies, because the over- or underestimation in the low energy region can influence the calibration and lead to a incorrect dose reading.

The readings of the described compact dosimeter layout with the PVC layer were tested at the CERN-EU highenergy Reference Field (CERF) facility [10] and can be found in Table 1. CERF is the facility providing neutron fields in a wide energy range typically produced by cosmic rays interacting with the atmosphere and by ions at

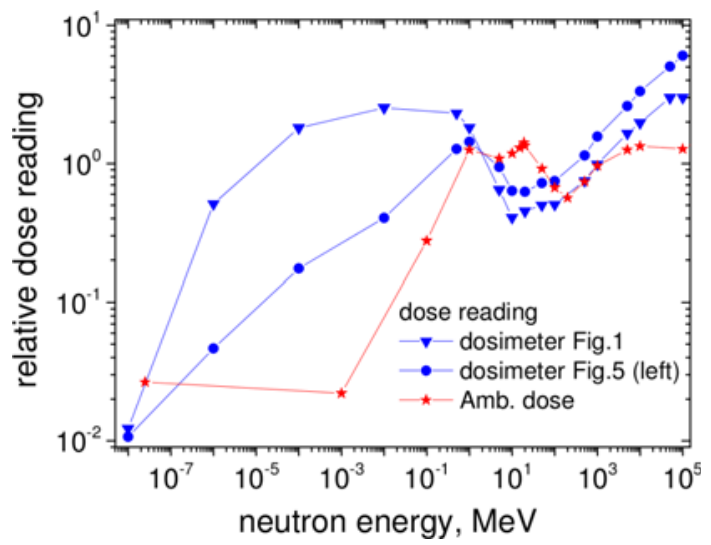

Figure 2. The relative dose readings for different dosimeter layouts from Fig. 1 and Fig. 5 and their comparison to the expected ambient dose. The values are normalized to the reading for the ${ }^{241} \mathrm{AmBe}$ source neutrons.

Table 1. The ratios of the measured at CERF ambient dose equivalent to the reference value and to the expected from FLUKA simulations with and without correction of the calibration to the scattered neutrons.

\begin{tabular}{cllll}
\hline $\begin{array}{c}\text { irradiation } \\
\text { direction }\end{array}$ & CERF & FLUKA & $\begin{array}{l}\text { CERF } \\
\text { corrected }\end{array}$ & $\begin{array}{l}\text { FLUKA } \\
\text { corrected }\end{array}$ \\
\hline front & $120 \%$ & $100 \%$ & $145 \%$ & $121 \%$ \\
\hline top & $129 \%$ & $109 \%$ & $155 \%$ & $131 \%$ \\
\hline
\end{tabular}

high energy accelerators. The first column 'CERF' are the ratios of the value measured with a dosimeter to the provided reference ambient dose equivalent value [11]. The measured value applied a calibration done with a ${ }^{241} \mathrm{AmBe}$ source under an assumption of only direct neutrons from the source with average fluence-to-dose conversion equal to $391 \mathrm{pSv} \cdot \mathrm{cm}^{2}$ mentioned in calculation section. Two values of a column are for the irradiation perpendicular to the TLD card surface and for the irradiation from the top of the dosimeter, both indicating angular dependence of the dosimeter response. Also to be mentioned is that the values in the table are the results averaged over several experimental runs with only slight deviations of few percent between the runs. In the second column 'FLUKA' are the ratios between the measured value and the one obtained folding the provided neutron spectrum during irradiation at the CERF facility and the calculated dosimeter response from simulations. The reference equivalent ambient dose value is a result of folding between the given spectrum and Pellicioni's fluence-to-dose coefficients. So the 'CERF' value shows the precision of the fluence-to-dose conversion of the dosimeter and the 'FLUKA' value shows the precision of the simulations. In the first approximation the dosimeter overestimated the $\mathrm{H}^{*}(10)$ value only about 20 $\%$. FLUKA simulations of the dosimeter response are defined to be $100 \%$ precise for the frontal irradiation.

The calibration of the neutron dosimeters, typically done using ${ }^{241} \mathrm{AmBe}$ or ${ }^{252} \mathrm{Cf}$ sources, requires the knowledge of the neutron spectrum, including the scattered neutrons. For this purpose a shadow cone technique [12] or 
simulations should be used. If the scattered neutrons contribute significantly to the neutron spectrum and are not taken into account the calibration can be underestimated and can provide lower measured values.

Another pair of columns in Table 1 marked as 'CERF corrected' and 'FLUKA corrected' are the ratios mentioned above, taking into account the experimentally verified neutron scattering.

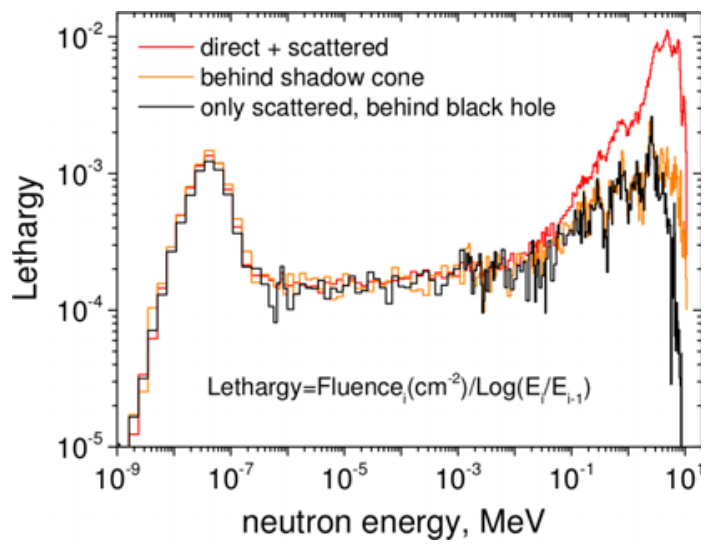

Figure 3. Simulated spectra of neutrons from the ${ }^{241} \mathrm{AmBe}$ source at the calibration point, behind the 'shadow cone' and behind the 'blackhole' providing only scattered neutrons.

The neutron scattering for the calibration procedure was simulated using the FLUKA code. The ${ }^{241} \mathrm{AmBe}$ source was positioned in FLUKA geometry inside the room, $1.6 \mathrm{~m}$ above the floor, $2.5 \mathrm{~m}$ below the ceiling, 3 $\mathrm{m}$ away from concrete walls in one direction and $6 \mathrm{~m}$ in another direction. To separate the scattered neutrons from those coming directly from the source so called 'blackhole' material was inserted into the FLUKA geometry between the source and the dosimeter. This FLUKA 'material' is introduced to stop the transport of particles and thus very suitable for our purposes. Instead of 'blackhole' in another simulation a paraffin wall was inserted with varying thickness. It was found that a $25 \mathrm{~cm}$ wall thickness is sufficient enough to stop the direct flux of neutrons from the source so having almost the same effect as the 'blackhole'. In Fig. 3 one can see the spectra for the described simulations. After folding the neutron spectra with and without the paraffin wall, or so called shadow cone, with Pellicioni's values it was found that the contribution from the scattered part to the dose is almost $20 \%$ of the initially expected. To test the simulation results the GSI ball dosimeter [13], with approved close to ideal response characteristics, was used. The measured readings of the dosimeter with and without the wall also showed 17 $\%$ difference for the doses absorbed by the TLD cards and thus around $17 \%$ difference for $\mathrm{H}^{*}(10)$ value, confirming the simulation results.

Coming back to the Table 1 , the $20 \%$ contribution to the equivalent ambient dose from the scattered neutrons, taken into account for calibration, changes the dosimeter reading. The ambient dose measured at CERF for the frontal irradiation is increased from $120 \%$ to $145 \%$. Still the $45 \%$ overestimation of the dose for the compact cylindrical dosimeter is a rather good agreement, which happens due to the good fluence-to-dose response in the region of interest for the accelerator complexes, i.e. thermal and from MeVs to few GeVs intervals [4]. Still the overestimation can be reduced if the response of the dosimeter will be improved in eVs-keVs energy region. The overestimation also occurs for the comparison between FLUKA simulations and measurements, if the scattered neutrons are taken into account. Thus our simulation instead of almost perfect fit shows a $20 \%$ overestimation for the calculated dose values, which is also quite acceptable and approves our simulation approach. As it is also clear from

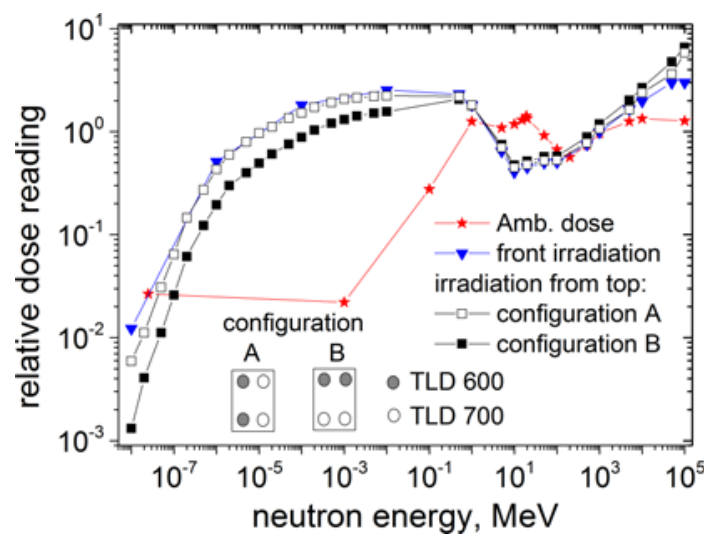

Figure 4. The relative dose readings of the compact $14 \mathrm{~cm}$ large dosimeter layout for irradiations from aside and from top for various configurations of TL chips in TLD card.

the table the response of the dosimeter is almost the same for irradiation from aside and from the top, despite the lack of the top and bottom caps of the lead cylinder. Thus the dosimeter read-out is quite angular independent though having only a cylindrical shape and rather small dimensions. The only requirement is set to the location of the chips in the card, they should be vertically the same in configuration A and not B as depicted in Fig. 4 with the responses for different irradiation directions and different TL chips configurations. For any irradiation perpendicular to the symmetry axis the readings are almost identical to the front irradiation and are independent on chips configuration. For the irradiation from top for the configuration $\mathrm{B}$ the material thickness pathed by neutron towards the TLD-600 chips is bigger and response of the dosimeter in low energy region decreases as it will be discussed below. In our measurements the configuration A was used and though the response for the irradiation from top is close to frontal irradiation (see Fig. 4) the simulations show bigger deviation from the measurements as can be seen in Table 1.

\section{Modification of the Andersson-Braun dosimeter}

A recently investigated layout showed that the compact size of the broad energy range neutron dosimeters is possible, but requires an improvement of the response in the 
low energy region. A solution could be a separation of the outer layer into 2 as in the modified Andersson-Braun type [14]. In Fig. 5 (left) one can see a schematic layout of a cylinder dosimeter, which has an outer $16 \mathrm{~cm}$ in diameter and $16 \mathrm{~cm}$ in height moderating high density polyethylene layer, afterwards a $10 \%$ borated $\mathrm{PE}$ or rubber to increase the absorption, then a lead layer and again a PE layer used to increase the response in the high energy region. In the middle of the dosimeter a TLD card is used

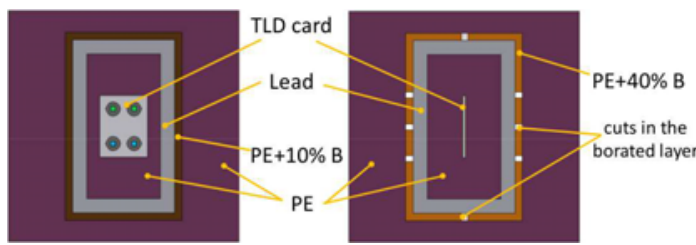

Figure 5. The modified Andersson-Braun dosimeter (left) and its further modification with concentric openings in the borated PE layer, side view relative to the TLD card (right).

as a sensitive element. Detailed description of the dimensions for the mentioned geometry and materials composition and densities can be found in [4]. The relative reading of the dosimeter can be found in Fig. 2 in comparison to the dosimeter from Fig. 1. The improved dose reading is clearly seen.

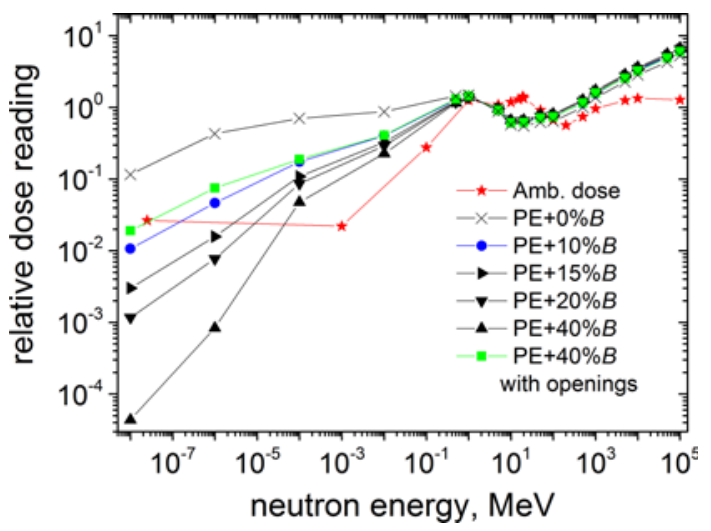

Figure 6. Dose readings of the dosimeter with varying borated polyethylene layer composition.

In Fig. 6 one can see the response of the dosimeter if the borated PE layer is modified. Using just a PE without any absorptive admixtures increases the response of the dosimeter at low and medium neutron energies, while an addition of $10 \%$ boron to the layer drastically improves the response. Further increase of the boron doping increases the underestimation of the dose at lower energies and for $40 \%$ of the mass the response at thermal energy is more than two orders of magnitude less than required. Following an idea in [15] to increase the response for low energy neutrons holes can be drilled in the dosimeter to provide more transparent flow of neutrons towards the TLD cards. Modifying the dosimeter with $40 \%$ doped PE layer as depicted in Fig. 5 (right), introducing concentric open- ings has the same influence as a decrease of the boron admixture.

\section{Modification of the compact dosimeter}

Another solution to improve the response of the compact dosimeter with a PVC layer could be changing the outer diameter of the dosimeter. For different neutron energies the relative ambient dose readings of the dosimeters with varying PVC layers can be seen in Fig. 7. For consistency in the figure the neutron energies are limited to $10 \mathrm{MeV}$, the red curve represents the expected values according to [7]. Decrease of the dimension leads to the reduced absorption and moderation, which makes it unfavourable to decrease further the size of the dosimeter. Still in high energy region the readings of more compact layouts are close to the one from Fig. 2 and thus even smaller dosimeters can be used for detection in the energy region above $1 \mathrm{MeV}$. The neutron spectra typically have a peak in the vicin-

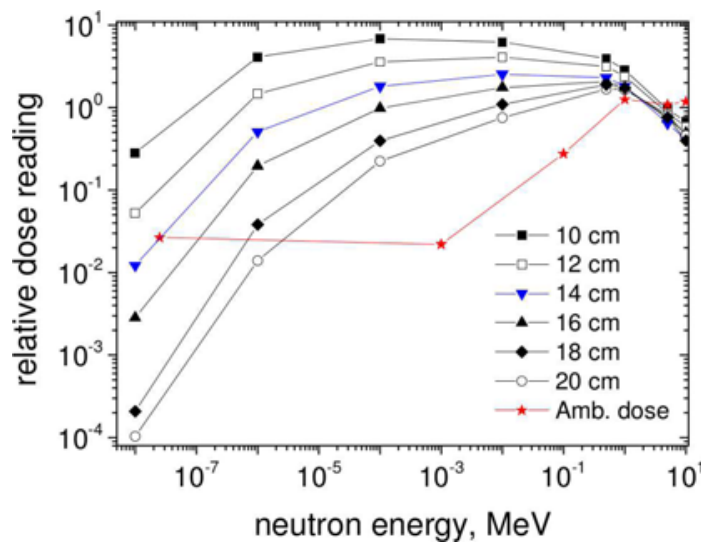

Figure 7. Readings of the dosimeter in low and medium energy region for different diameters of the PVC layer from $10 \mathrm{~cm}$ to 20 $\mathrm{cm}$.

ity of thermal energies requiring an appropriate reading around $25 \mathrm{meV}$. A diameter of $14 \mathrm{~cm}$ of the current compact PVC cylinder is optimal for such energies. Increase of the dosimeter dimension bends the response curve in such a way that the dose reading is underestimated around 25 $\mathrm{meV}$ and for eVs-keVs region it is better due to more efficient moderation. For the dosimeter with the diameter and height of $20 \mathrm{~cm}$ the reading in the thermal region is almost two orders of magnitude smaller due to strong absorption and thus not acceptable, though in eVs-keVs region the reading is drastically improved in comparison to the $14 \mathrm{~cm}$ layout. Following our investigations with the dosimeter layout from Fig. 5 the openings were cut through the PVC layer. In Fig. 8 the simulated relative readings are shown when additional openings $1 \mathrm{~mm}$ and $0.5 \mathrm{~mm}$ in width are introduced in PVC layer, as depicted in the insertion of the figure. Such dosimeters would have very angular dependent response and are not usable in practice.

Extensive simulations were performed in order to investigate the influence of different openings configurations, their widths and depths. Finally it was found that an 


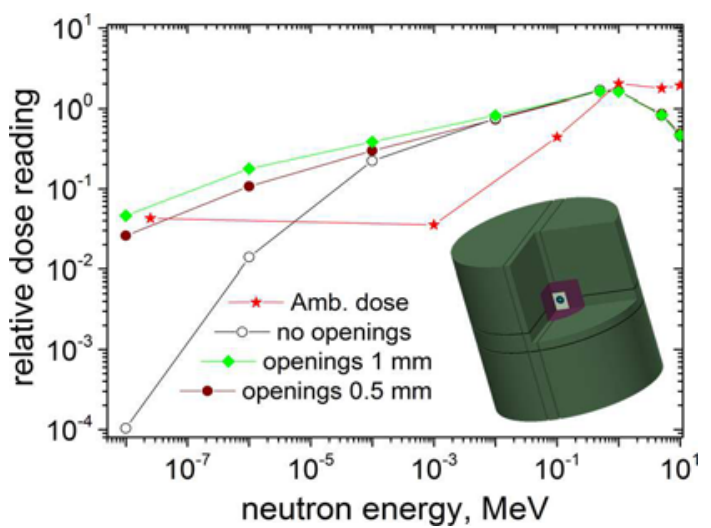

Figure 8. Responses of dosimeters having $20 \mathrm{~cm}$ diameter with and without openings and comparison to the required ambient dose values. Insertion shows the layout with $1 \mathrm{~mm}$ wide openings.

optimal configuration providing nearly angular independent reading is achieved for a dosimeter layout shown on Fig. 9. The initial $6.7 \mathrm{~cm}$ in diameter (d) and $7.1 \mathrm{~cm}$ in

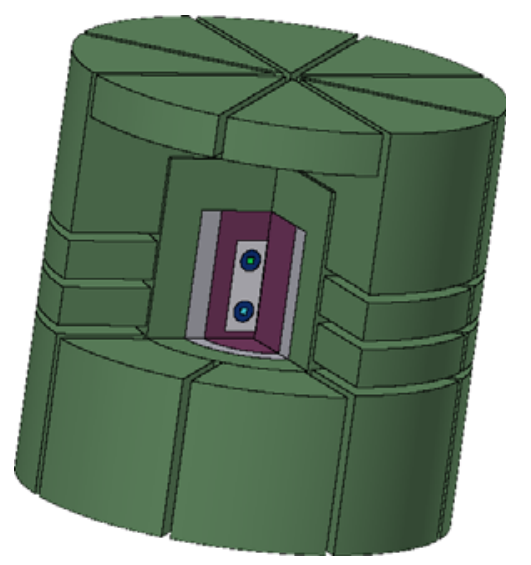

Figure 9. Neutron dosimeter in 3D view (obtained using Flair [6]) with openings in PVC layer. Material colours correspond to those in Fig. 1.

height (h) lead cylinder containing high density polyethylene cylinder $(\mathrm{d}=4.7 \mathrm{~cm}$ and $\mathrm{h}=7.1 \mathrm{~cm})$ is located in futuristic structure of PVC layer cut to a depth of $5.5 \mathrm{~cm}$ from the side and to $5 \mathrm{~cm}$ from the top and bottom with openings, each $2 \mathrm{~mm}$ in width. The openings release some of material making the dosimeter up to $0.5 \mathrm{~kg}$ lighter. The inner PVC part and openings can be varied in such a way that increase of the openings width or their amount can be compensated with the increase of the non-cut PVC region.

\section{Summary and conclusion}

The final neutron dosimeter layout shown in Fig. 9 improves the fluence-to-ambient dose conversion and provides almost angular independent equivalent dose reading. In Fig. 10 one can find the simulated reading of the dosimeter in wide energy range, green curve with square symbols. Folding the response of the dosimeter from Fig. 9 with the CERF spectrum should provide almost $100 \%$ fit with the expected equivalent ambient dose value. Taking into account our comparison between FLUKA simulations and measurements would most probably lead to a dose overestimation of around $20 \%$. The

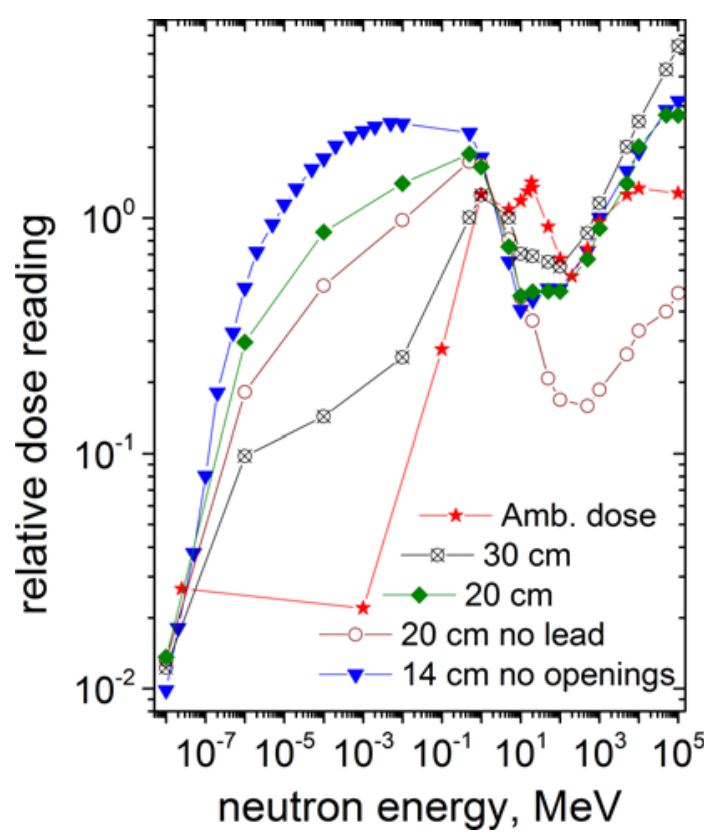

Figure 10. Readings for the dosimeters: (green curve) layout from Fig. 9; (brown curve) the same layout with PE instead of lead; (blue curve) layout from Fig. 1; (black curve) example of a dosimeter with $\mathrm{d}=\mathrm{h}=30 \mathrm{~cm}$; (red curve) expected ambient dose equivalent.

dosimeter can be further increased in dimensions and provided with the openings will have even more improved reading, example of such dosimeter response can be also found in Fig. 10, black curve for a $30 \mathrm{~cm}$ diameter dosimeter, though such a dosimeter is already quite bulky, heavy and complicated for production. Additionally a reading for the dosimeter with the same layout as in Fig. 9, but with a polyethylene instead of lead, is also depicted in Fig. 10 and provides even better response for low and medium energies.

The final design from Fig. 9 consumes an appropriate response from the initial design in high energy neutron fields and improves it for medium energies. Thus the dosimeter response is covering almost 12 orders of magnitude of neutron energies and is rather sufficient for the usage at high energy accelerators.

\section{References}

[1] https://www.gsi.de ; https://www.fair-center.de.

[2] M. Awschalom and R. S. Sanna, Applications of Bonner sphere detectors in neutron field dosimetry, Radiation Protection Dosimetry, Vol.10 No. 1-4 pp. 89-101 (1985).

[3] G. Fehrenbacher, F. Gutermuth, E. Kozlova, T. Radon and R. Schuetz, Neutron dose measurements with the 
GSI ball at high-energy accelerators, Radiation Protection Dosimetry (2007), Advance Access publication 16 September 2007, Vol. 125, No. 1-4, pp. 209-212.

[4] A. Sokolov, G. Fehrenbacher, T. Radon , Design Development of a passive neutron dosemeter for the use at high-energy accelerators, Radiation Protection Dosimetry 2015; doi: 10.1093/rpd/ncv489

[5] A. Fasso“, A. Ferrari, J. Ranft and P. R Sala, FLUKA: a multi-particle transport code, CERN-2005-10 (2005), INFN/TC 05/11, SLAC-R-773.

[6] V. Vlachoudis, FLAIR: A Powerful But User Friendly Graphical Interface For FLUKA Journal Name, Saratoga Springs, New York, 2009.

[7] M. Pelliccioni, Radiation Protection Dosimetry, Nuclear Technology Publishing, 88, 279-297 (2000).

[8] ISO, International Standardization Organization, Neutron Reference Radiations for Calibrating Neutron Measurement Devices Used for Radiation ProtectionPurposes. ISO 8529, 1st Edition, 1989.

[9] I.A. Zahradnik, Development of the passive neutron dosimeter, ANDREA, for high energy accelerators, verification measurements and simulation of the dose response using the Monte Carlo code , bachelor thesis,
THM Giessen, 2015.

[10] A. Mitaroff and M. Silari, The CERN-EU highenergy Reference Field (CERF) facility for dosimetry at commercial flight altitudes and in space, Radiation Protection Dosimetry 102, 7-22, 2002.

[11] http://cerf-dev.web.cern.ch/content/fluka-referencevalues.

[12] H. Kluge, A.V. Alevra, S. Jetzke, K. Knauf, M.Matzke, K. Weise and J. Wittstock, Scattered neutron reference fields produced by radionuclide sources, Radiation Protection Dosimetery, Nuclear Technology Publishing, 70, Nos. 1-4, pp. 327-330 (1997).

[13] F. Gutermuth, T. Radon, G. Fehrenbacher and J.G. Festag, The response of various neutron dosemeters considering the application at a high energy particle accelerator. Kerntechnik 68, 172-179 (2003).

[14] V. Mares, A.V. Sannikov and H. Schraube, Response functions of the Andersson-Braun and extended range rem counters for neutron energies from thermal to 10 $\mathrm{GeV}$, Nuclear Instruments and Methods in Physics , Vol. 476, Issues 1-2, 1 Jan 2002, p. 341-346.

[15] F. H. Attix (Editor), Topics in Radiation Dosimetery: Radiation Dosimetery, Band 1, Academic Press 1972 